\title{
Detectivity comparison of bolometric optical antennas
}

\author{
Alexander Cuadrado ${ }^{a, b}$, José M. López-Alonso ${ }^{a}$, Juan C. Martínez- Antón ${ }^{a}$, \\ José M. Ezquerro ${ }^{a}$, Francisco J. González ${ }^{c}$, and Javier Alda ${ }^{a}$ \\ ${ }^{a}$ Applied Optics Complutense Group, University Complutense of Madrid, \\ Ave. Arcos de Jalón, 118. 28037 Madrid. Spain \\ ${ }^{b}$ Laser Processing Group. Institute of Optics. Consejo Superior de Investigaciones Científicas \\ Serrano 121. 28006 Madrid. Spain. \\ ${ }^{c}$ Coordinación para la Innovación y la Aplicación de la Ciencia y la Tecnología, Universidad \\ Autónoma de San Luis Potosí, Sierra Leona 550, Lomas 2a Sección, SLP, Mexico
}

\begin{abstract}
The practical application of optical antennas in detection devices strongly depends on its ability to produce an acceptable signal-to-noise ratio for the given task. It is known that, due to the intrinsic problems arising from its sub-wavelength dimensions, optical antennas produce very small signals. The quality of these signals depends on the involved transduction mechanism. The contribution of different types of noise should be adapted to the transducer and to the signal extraction regime. Once noise is evaluated and measured, the specific detectivity, $D^{*}$, becomes the parameter of interest when comparing the performance of antenna coupled devices with other detectors. However, this parameter involves some magnitudes that can be defined in several ways for optical antennas. In this contribution we are interested in the evaluation and comparison of $D^{*}$ values for several bolometric optical antennas working in the infrared and involving two materials. At the same time, some material and geometrical parameters involved in the definition of noise and detectivity will be discussed to analyze the suitability of $D^{*}$ to properly account for the performance of optical antennas.
\end{abstract}

Keywords: detectivity, optical antennas, bolometers, infrared detectors, noise, figures of merit

\section{INTRODUCTION}

The interaction of light with metallic sub-wavelength structures has opened the way to new applications and ideas for developing nanophotonics elements. ${ }^{1-3}$ Resonant structures may work connected to an external circuit, or by themselves, modifying the transmitted, absorbed, or reflected spectra, ${ }^{4}$ the polarization state of an incoming beam, ${ }^{5}$ or even the phase. ${ }^{6}$ Joining all these possibilities we may think of new devices in the so called resonant optics arena. ${ }^{7,8}$ Actually, these elements working in the optical range were already present at lower frequencies, as frequency selective surfaces,${ }^{9}$ wire and meanderline polarizers, ${ }^{10}$ and reflectarrays. ${ }^{11}$ On the other hand, these antenna-like structures can be connected to an external circuit to provide an electrical signal proportional to the irradiance falling on the antenna. In this case, besides the resonant structure, they require a transduction mechanism able to convert the optical power in an electric magnitude. These elements actually work as receiving optical antennas, sharing with their radioelectric counterparts the same kind of characterization and parameterization.

However, optical antennas do not responds to radiation as radioelectric or microwave antennas. Metals at optical frequencies are dissipative, and plasmonic resonances appear elsewhere. On the other hand, optical antennas working as light detectors are exotic and should be handled with caution when applying the same kind of characterization parameters used for other detection technologies. Most of figures of merit for optical detectors are well suited for detectors having a size larger, or much larger, than wavelength, and involving charge carrier transitions between bands. On the contrary, optical antennas are small, subwavlength, present polarization selectivity, and interact with light almost classically. This is a consequence of the antenna-like elements of the

Further author information: (Send correspondence to Javier Alda)

Javier Alda: E-mail: javier.alda@ucm.es, Telephone: +34.91.3946874

Plasmonics: Metallic Nanostructures and Their Optical Properties XIII, edited by Allan D. Boardman, Din Ping Tsai, Proc. of SPIE Vol. 9547, 954735 - (c) 2015 SPIE · CCC code: 0277-786X/15/\$18 · doi: 10.1117/12.2187926 
Table 1. Values of responsivity $(\mathrm{V} / \mathrm{W})$ for dipole antennas coupled to waveguides, extracted from reference $\mathrm{e}^{15}$ as a function of the angle between the dipole and the electric field.

\begin{tabular}{|c|c|}
\hline Polarization angle $\left(^{\circ}\right)$ & Responsivity $(\mathrm{V} / \mathrm{W})$ \\
\hline 0 & 0.0116 \\
\hline 10 & 0.0113 \\
\hline 20 & 0.0104 \\
\hline 30 & 0.0101 \\
\hline 40 & 0.0102 \\
\hline 50 & 0.0099 \\
\hline
\end{tabular}

device. Besides, to properly produce a feasible signal, optical antennas require transduction mechanisms. At this point, we may distinguish three demonstrated physical mechanism: optical rectification using metal-oxide-metal junctions ${ }^{12}{ }^{12}$ bolometer effect, ${ }^{13}$ and thermoelectric transduction. ${ }^{14}$ They work quite differently and show special characteristics associated with its intrinsic behavior.

In this contribution we will discuss how detectivity is affected by the special characteristics of optical antennas. To do that, we first present in section 2 those figures of merit that best apply to our case. Section 3 is devoted to the description of the main noise sources applicable to optical antennas. Responsivity is discussed in section 4 and the main findings of the paper are summarized in section 5 .

\section{FIGURES OF MERIT FOR OPTICAL ANTENNAS}

When considering the applicability of a given detection technology to a given detection situation we should consider several aspects. In this section we describe some applicable figures of merit or meaningful parameters.

- Responsivity, $\mathcal{R}$. This is probably one of the most relevant figures of merit because it establishes the kind of signal that the detector is producing. It is defined as the ratio between the signal parameter (voltage or current) and the power that arrives to the detector. The level of the responsivity is a key factor to adapt the signal conditioning and amplification electronics. For optical antennas, responsivity is measured in terms of $\mathrm{V} / \mathrm{W}, \mathcal{R}_{\mathcal{V}}$, or $\mathrm{A} / \mathrm{W}, \mathcal{R}_{\mathcal{I}}$, depending on the biasing strategy.

$$
\mathcal{R}_{V}=\frac{V}{P}, \quad \text { or } \quad \mathcal{R}_{I}=\frac{I}{P},
$$

where $P$ is the incident power on the antenna, and $V$ or $I$ are the voltage or current signal. The actual value for optical antennas is quite low and is about $1 \mathrm{~V} / \mathrm{W}$ for metal-oxide-metal systems. Some other type of antennas based on bolometric effect have been measured and the reported values of responsivity as a function of the angle of polarization of the incident electric field are presented in table $1^{15}$ (the values of this table is well below the $1 \mathrm{~V} / \mathrm{W}$ for metal-oxide-metal transducers because they are bolometric in nature and the bolometer is located at the end of a metal waveguide).

- Time of response, $\tau$. This parameters also defines the bandwidth of the detector. A simple, but useful relation between bandwidth and time response is $\Delta f=1 / 2 \tau$, being $\tau$ the characteristic time of the temporal response of the detector. Depending on the transduction mechanism the detector may respond in very short times. Band transitions associated with semiconductor technology are usually very fast. For optical antennas, metal-oxide-metal transductors are, by far, the fastest devices, theoretically reaching the picosecond range. ${ }^{12}$ If bolometric or Seebeck effect are the transduction mechanism, its thermal character make them slower than metal-oxide-metal rectifiers and the calculated response time may reach the microsecond range, ${ }^{16}$ although a conservative evaluation place the characteristic time or response in few microseconds (see figure 1).

- Detection area, $A_{d}$. This parameter describes the spatial extension of detector. Its definition is very well suited for those detector having a detection area much larger than wavelength. This is the case of most of 


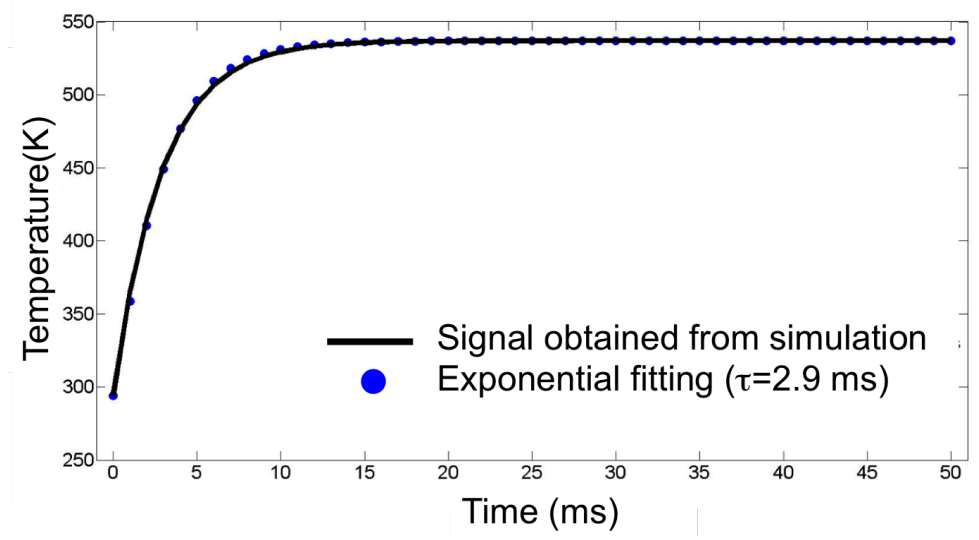

Figure 1. Temporal evolution of temperature for a $2.8 \mu \mathrm{m} \mathrm{Au}$ dipole placed on a $\mathrm{Si} / \mathrm{SiO}_{2}$ substrate and receiving a plane wave linearly polarized along the direction of the dipole. The electric field amplitude is $220 \mathrm{~V} / \mathrm{m}$. The dots corresponds with an exponential fitting of the type $T(t)=T_{s}+\left(T_{0}-T_{s}\right) \exp \left(-\frac{t}{\tau}\right)$, where $T_{s}$ is the steady-state temperature, and $T_{0}$ is room temperature. The time constant of the exponential is $\tau=2.9 \mathrm{~ms}$.

semiconductor detectors. However, when considering optical antennas we should recall that they are, in nature, smaller than wavelength and can be considered as the smallest possible optical detector. ${ }^{17}$ Actually, spatial responsivity map measurement requires a careful preparation of the probing beam and a precise scanning of it over the detector. Then, the obtained signal has to be deconvolved to obtain the desired result (see figure 2). From a computational point of view, the evaluation of the spatial response can be done by considering the antenna as an emitting device. The map of the electric field at the plane of the antenna provides an adequate representation of the spatial responsivity. ${ }^{18}$ Once the spatial responsivity map is obtained, the actual value of the detection area is defined as comprising a given amount of the total spatial responsivity. A $50 \%$ or a $90 \%$ criterion has been used in the past to obtain this parameter. It means that the given percentage of the total spatial response is included within a perimeter that encircles the desired detection area. Then, the $90 \%$ criterion provides a larger detection area than the $50 \%$. We may notice that some arbitrary choice of the collected percentage can vary the value of the detection area. Taking into account these definitions we can calculate the detection area that is typically around $A_{d}=\lambda$ (being $A_{d}$ in units of area). This problem is specific of this type of detectors where the size is subwalenght in nature. Therefore, a quite straightforward solution to circumvent this situation is to use the actual physical exposed area of the antenna. In this case, the value of the detection area of optical antenna becomes very small and could be below $A_{d}=10^{-3} \lambda^{2}$. We will discuss this problem when evaluating detectivity in section 4.

Although the previous definition of the detection area can be realized and used, there is still another interesting aspect that should be taken into account and it is also specific of optical antennas. In reference ${ }^{15}$ another spatial parameter, $N$, is included in the discussion:

$$
N=\frac{|\langle\Phi \mid \Psi\rangle|^{2}}{\langle\Phi \mid \Phi\rangle\langle\Psi \mid \Psi\rangle}
$$

where $\Phi$ is the detector spatial response map in amplitude, $\Psi$ is the electric field distribution of the incident beam, and $\langle\Phi \mid \Psi\rangle=\iint \Phi(x, y) \Psi^{*}(x, y) d x d y$, represents the maximum overlapping between these two maps. This parameter describes how the spatial amplitude distribution of a given laser beam is interacting with the response of the antenna (in amplitude, not in irradiance). Some practical difficulties appear when realizing this parameter. From an experimental point of view it requires the knowledge of the response of the antenna in amplitude that can be obtained from near-field optical scanning microscopy techniques. ${ }^{20}$ On the other hand, computational electromagnetism package provide the values of the electric field. By including this parameter in the definition of the detection area, we are incorporating the effect of the electromagnetic coupling of the spatial modes of the incident beam with the detection modes of the optical 

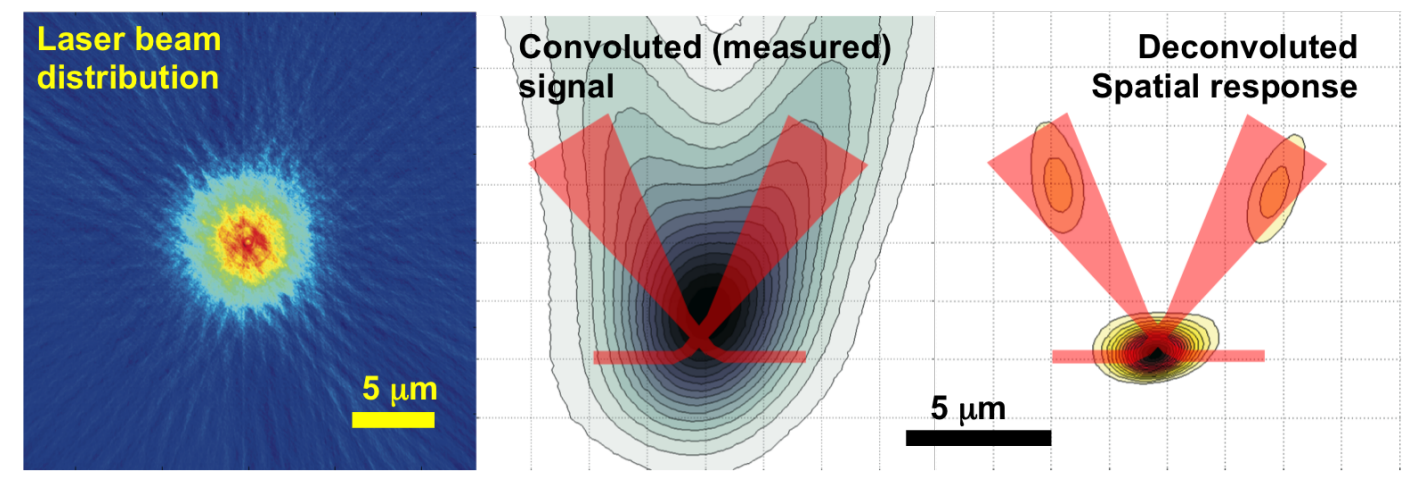

Figure 2. The map of the spatial response is obtained by scanning a known irradiance (left) on the plane of the antenna. The measured signal is the convolution of the actual spatial response with the laser irradiance (center). The spatial response is obtained after deconvolving the measured signal with the map of the irradiance of the incident beam (right). These plots were obtained for a dipole antenna excited by a visible laser beam at $655 \mathrm{~nm}$. The shape of the antenna is schematically added to the measured and deconvoluted spatial response. The lobes of the spatial response correspond with the signal extraction lines of the device. The polarization is horizontal and aligned with the dipole (extracted from $\operatorname{ref}^{19}$ )

antenna. Figure 3 show how the electric field around the antenna follows a characteristic pattern when antenna is considered in emission mode. For an optimum fitting between the input electric field and the spatial responsivity map (in amplitude) the incident beam should adapt to the specific spatial pattern of the antenna.

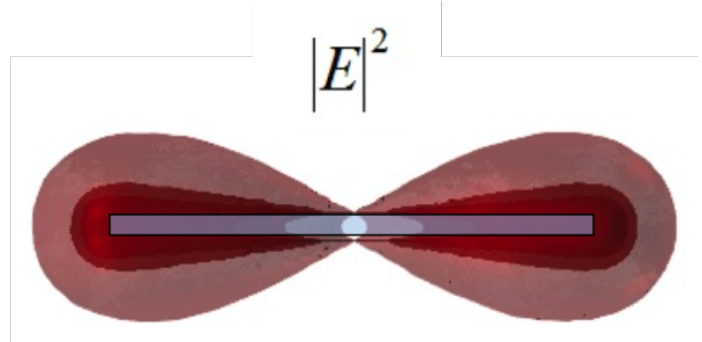

Figure 3. Map of the square modulus of the electric field at the near-field zone of a dipole antenna.

- Signal to Noise Ratio, SNR. This ratio represent how many times the signal is larger than the noise. It is a dimensionless parameter and it can be obtained as the quotient of the mean value of the signal divided by its standard deviation. It also can be expressed in terms of the responsivity as: $\mathrm{SNR}=\frac{\mathcal{R} P}{V_{n}}$, where $P$ is the power radiant flux (in watts), and $\mathcal{R}$ and $V_{n}$ are the Responsivity (in volts per watts), and the noise voltage (in volts) respectively. In this previous discussion we have considered a system that provides a voltage signal. If the signal is an electric current, a similar dependence can be given using the associated magnitudes in amps.

- Noise Equivalent Power, NEP. It can be defined as the optical power that produces a SNR $=1$. Therefore it could be given as: NEP $=\frac{V_{n}}{\mathcal{R}}$. This parameter is strongly dependent on the transduction mechanism used for detecting light, and the environment and auxiliary elements around the detector. The complete treatment of noise is the topic of dedicated books and chapters of books and here we are only considering those elements more relevant in optical antennas. ${ }^{21}$ 
- Detectivity. This parameter is used for comparing detection technologies and products. It is defined as

$$
D^{*}=\frac{\sqrt{A_{d} \Delta f}}{\mathrm{NEP}},
$$

where $A_{d}$ is the detection area, $\Delta f$ is the detector bandwidth, and NEP is the Noise Equivalent Power. These parameters have been previously defined and discussed. Section 4 is devoted to the analysis of this parameter.

Detectivity can be measured following successive steps. The first one is the measurement of responsivity, that, for our purposes is defined as

$$
\mathcal{R}_{V}=\frac{d V}{d P}
$$

that is the incremental version of Eq. (1). As we have previously see, $V$ is the DC voltage given by the device (or the intensity current if this magnitude is measured instead of voltage), and $P$ is the optical power delivered to the antenna. This definition of responsivity is well suited in case some radiometric inaccuracies are present. The most precise way of determining the value of $\mathcal{R}_{V}$ is to acquire several pairs or voltage versus power, fit these points to a polynomial curve (normally linear) and calculate the derivative at each point. Usually, the fitted dependence is linear, and responsivity can be seen as the slope of the fitted curve. In order to do the previous procedure, a large number of values of voltage and power, $\left(V_{i}, P_{i}\right)$, are usually taken at each point. Then, it is possible to obtain a mean value and a RMS value of voltage for different incident power. In this sense, a Noise Equivalent Power (NEP), can be defined as

$$
\mathrm{NEP}=\frac{\sigma_{V}}{\mathcal{R}_{V}}
$$

being $\sigma_{V}$ the RMS value of the voltage. Normalizing this value with the detector area and the detector bandwidth discussed above, detectivity can be obtained from equation (3). One remaining question is how to calculate the power actually delivered to the antenna. This number is very important in order to calculate the responsivity because of equations (1) and (2). From these equations is clear that, in case of optical and infrared antennas, the magnitude or power sent to the antenna is not the power delivered to the antenna, because the matching among the incoming radiation modes and the absorption modes of the antenna is a factor to be considered. This is a point that is not taken into account when trying to translate the measure of detectivity from semiconductor detectors to antennas.

\subsection{Measuring set-up}

The characteristics parameters defined in this section can be measured using a dedicated experimental set-up. The system consist in several independent modules that need to be properly adjusted and combined. Typically, the light source is a laser or a collection of lasers to perform a multispectral analysis. The output beam is monitored to consider temporal fluctuations of the irradiance. This monitoring also serves to known the power delivered to the antenna and then calculate $\mathcal{R}$. The laser beam is modulated to avoid contributions from $1 / f$ noise. Also the polarization state of the beam can be change by the appropriate polarizers and retarders. Using them, a collection of polarization states are delivered to the optical antenna to perform a complete characterization. Optical antennas are attached a $X Y Z$ stage to place them at the right distance where the waist of the laser beam is placed. This means that the mode of the beam has to be as Gaussian as possible to assure a plane wavefront at the the measurement plane. Besides, the antenna is moved under the beam using the stage. The focusing optics of the system should be fast enough to conform a small laser spot when performing spatial responsivity measurement. For the rest of measurements, the constrains are not so tight and the focusing element can be slower. The time of response is measured by changing the modulation frequency and registering the extinction of the signal when temporal frequency go beyond the bandwidth of the element. The signal from the antenna is sent to a lock-in amplifier that receives the synchronizing signal from the modulation. Usually, before arriving to the lock-in amplifier, the signal is amplyfied and DC-filtered using a low-noise circuitry. All the system should be controlled by computer to produce more reliable results. An schematic view of the measuring set-up is depicted in figure 4

Proc. of SPIE Vol. $9547954735-5$ 


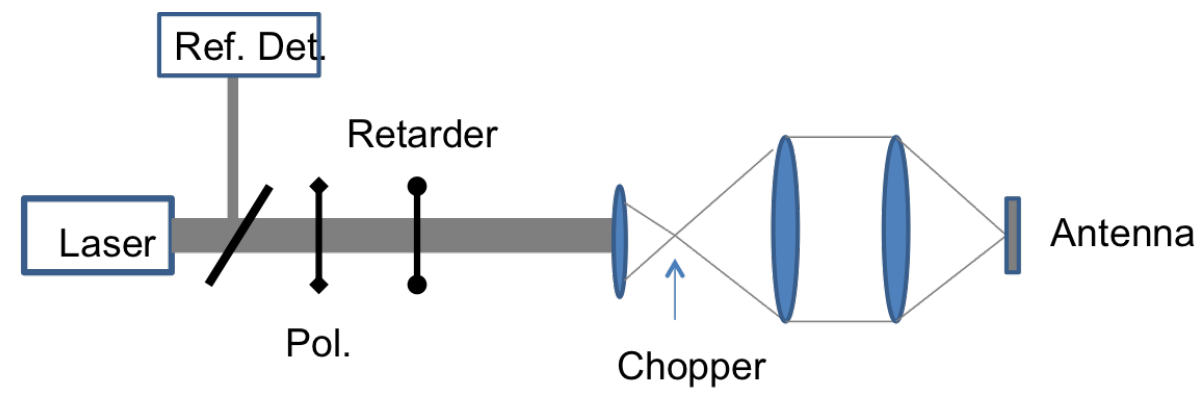

Figure 4. Experimental set-up used for the measurement of the characteristics parameters of an optical antenna working as a light detector. The antenna is placed on a moving stage to have it scanned under the proving beam. In case angular measurements are necessary, the antenna should be placed on a goniometric stage.

\section{NOISE IN OPTICAL ANTENNAS}

When considering optical antennas as light detectors we can choose different transduction mechanisms as they were described in section 1. In this contribution we will focus our attention to optical antennas using thermal mechanism: bolometric or thermoelectric. These elements work when optical radiation induce currents in the resonant element and these currents dissipate power that generates a change in temperature. In bolometric devices this change in temperature modifies the voltage drop along the device, and in thermoelectric devices the active resonant element antenna is located at the hot junction of a thermocouple.

In this section we collect the noise contributions that are applicable to optical antennas that use the bolometric effect. Due to the classical character of the bolometric transduction mechanism, contributions from photon noise or generation-recombination noise are not applicable.

- Johnson Noise. For these devices we have computed the Johnson noise that corresponds with the following NEP

$$
\mathrm{NEP}_{\text {Johnson }}=4 k_{B} T_{d} \Delta f
$$

where $k_{B}$ is the Boltzman constant, and $T_{d}$ is the temperature of the device. At this point we should note that optical antennas present a temperature map that is related with the dissipated power at the antenna location. Therefore, $T_{d}$, should be taken here as a mean temperature of the antenna.

- Thermal noise. Another noise contribution is coming from the radiative heat exchange between the device, at a temperature $T_{d}$, and the surroundings, at a temperature of $T_{0}$. A simple approach is consider the detector as a grey body, characterized by an emissivity, $\epsilon$, and the surrounding as a black body. In this case, the NEP is given as: ${ }^{21}$

$$
\mathrm{NEP}_{\text {therm }}=\sqrt{\frac{8 k_{B} A_{d} \sigma_{\mathrm{SB}} \Delta f\left(T_{d}^{5}+T_{0}^{5}\right)}{\epsilon}}
$$

where $\sigma_{\mathrm{SB}}$ is the Stefan-Boltzman constant, and $\epsilon$ is the emissivity of the device.

- Temperature noise. Meanwhile the previous noise source describes the radiative balance between detector and environment, This temperature noise contribution takes into account the change in temperature due to charge carrier transport and conduction mechanisms. Bolometric optical antennas provide a signal that is given as

$$
V_{\text {signal }}=V_{b} \alpha \Delta T
$$

where $V_{b}$ is the bias voltage, $\alpha$ is the Temperature Coefficient of Resistance of the metal, and $\Delta T$ is the change in temperature produced by the optical irradiance and its radiative exchange. These devices are affected by a temperature noise ${ }^{21}$ that can be converted to a NEP as

$$
\mathrm{NEP}_{\text {temp }}=V_{b}^{2} \alpha^{2} T_{d}^{2} \frac{4 K k_{B} \Delta f}{K^{2}+4 \pi^{2} f^{2} C^{2}},
$$


where $f$ is the modulation frequency, $C$ is the heat capacity of the device (it is calculated using the specific heat capacity and the mass of the device), and $K$ is the thermal conductance of the device. This thermal conductance depends on the thermal conductivity and the geometry of the antenna as:

$$
K=\kappa \frac{S}{L},
$$

being $\kappa$ the thermal conductivity, $S$ is the section of the device where the heat flows through, and $L$ is the length of the heat flow within the device. This parameter is strongly dependent on the geometry of the system and the arrangement of auxiliary elements as the load lines.

- Thermistor noise. Optical antennas working as bolometric detectors are included in a read-out electronic circuit that can take several forms. The simplest one is to place the antenna within a voltage divider. The current from the bias source flows through two resistances: the load resistance, $R_{L}$, and the detector resistance, $R_{d}$. Then, this noise contribution takes into account this situation. Actually, temperature noise described above is very much related with the thermistor noise in bolometers ${ }^{21}$ that generates the following expression for NEP

$$
\mathrm{NEP}_{\text {thermistor }}=\frac{1}{\alpha \epsilon} \sqrt{4 k_{B} T_{d} R_{d} \Delta f\left(K_{\text {eff }}^{2}+4 \pi^{2} f^{2} C^{2}\right)},
$$

being $R_{d}$ the resistance of the detector (the typical value for bolometric optical antennas is around $100 \Omega$ ), and $K_{\text {eff }}$ is an effective conductance that is equal to the conductance of the device (see Eq. (10)) when the load and the device have the same resistance, $R_{L}=R_{d}$.

Both temperature and thermistor noises (equations (9) and (11)) depend on the frequency of modulation of the signal, $f$. As a practical approach we could make this frequency equal to zero to obtain a DC noise contribution. In this case, the thermal inertia of the device is not taken into account.

Once these contributions are considered they should be added in quadrature to generate a total NEP for the given device. When defining the previous noise contribution we have already seen that some parameters cannot be accurately defined for optical antennas: $T_{d}$ is a map; subwavelength elements are difficult to model with a given grey body emissivity, $\epsilon$; and thermal conductance requires heat flow lines be considered from and to the external load lines and substrate. Therefore, all these questions and uncertainties will influence the detectivity value.

\section{DETECTIVITY}

Equation (3) describes this parameter as proportional to the square root of the area of detection, $A A_{d}$ and bandwidth $\Delta f$, and inversely proportional to NEP. Using this reasoning we may understand that faster, larger, and less noisy detectors are desirable. However, for optical antennas it is not clear why the small detection area of these devices plays against optical antennas when compared with other detection technologies using $D^{*}$.

In this section we have considered two dipoles that have been simulated using Comsol Multiphysics to know their thermal and electromagnetic performance. The results obtained from the simulation are included in the noise models presented in section 3. Both optical antennas are dipoles crossed with a load line. The difference between them is the material of the dipole: gold and titanium. The load lines are made of the same material than the dipole. The operation wavelength is $\lambda_{0}=10.6 \mu \mathrm{m}$. We also assumed that light is modulated at a frequency $f=1 \mathrm{Khz}$. From simulation we have also calculated the change in the dipole length ${ }^{22}$ due to the different material characteristics. The values used for the time of response of the dipole are $2.9 \mathrm{~ms}$ for the Au dipole and $3.5 \mathrm{~ms}$ for the Ti. The value of the resistance has been evaluated using the electric resistivity of the materials and the geometry of the dipoles. The area of detection has been calculated by two means: $A_{d 1}=\frac{\pi}{4}(l+\lambda)(w+\lambda)$, where $l$ is the length of the dipole, $w$ is the width, and $\lambda$ is the effective wavelength defined as $\lambda=\lambda_{0} / \sqrt{\left(\varepsilon_{\mathrm{Si}}+\varepsilon_{\text {air }}\right) / 2} \cdot{ }^{23}$ The other calculated area is the geometrical section exposed to the incoming radiation, $A_{d 2}=l w$. The results are presented in table 2. They show that Ti dipoles present a better $D^{*}$ than Au dipoles. This is in accordance with previous results where titanium optimized devices provided larger signals. ${ }^{22} D^{*}$ is also larger when considering a larger detection area, $A_{d}=A_{d 1}$. At the same time we may see that $D^{*}$ values are not very competitive with 
Table 2. Values of $D^{*}$ for two dipoles made of $\mathrm{Au}$ and Ti, considering two different values of the detection area. The units for $D^{*}$ are $[\mathrm{cm}][\mathrm{Hz}]^{1 / 2}[\mathrm{~W}]^{-1}$

\begin{tabular}{|c|c|c|}
\hline & $\mathrm{Au}$ & $\mathrm{Ti}$ \\
\hline$A_{d 1}$ & $9.08 \times 10^{5}$ & $1.32 \times 10^{8}$ \\
\hline$A_{d 2}$ & $8.34 \times 10^{6}$ & $1.21 \times 10^{9}$ \\
\hline
\end{tabular}

actual detection technologies in the infrared. On the other hand, the values obtained here for $D^{*}$ are still few orders of magnitude larger than those obtained experimentally. This discrepancy should be addressed by a more detailed analysis of the actual values of the magnitudes involved in the definition of NEP and detectivity.

Along this evaluation we have calculated the NEP contributions. The dominant noise processes have been thermal and thermistor noises $\mathrm{NEP}_{\text {therm}}$, being $\mathrm{NEP}_{\text {temp }}$ the less important.

\section{CONCLUSIONS}

In this contribution we have analyzed the suitability of current figures of merit, specially detectivity, $D^{*}$, as good descriptors for optical antennas. One of the main drawbacks of $D^{*}$ to describe optical antennas is its dependence on the area of detection, $A_{d}$, that is one of the main advantages of optical antennas when compared with some other detection technologies. Even the definition of this area of detection should be open to discussion. Noise contributions have been restricted to those that are more important for optical antennas. The numerical results show that thermal and thermistor noises are the most contributing processes, being temperature noise the less important.

Summarizing the results of this paper we could see that detectivity is a figure of merit that adapt poorly to optical antennas because of the difficulties to establish a well defined detection area. Even more, the detection area should be considered in conjunction with the good overlapping of the incoming field distribution. On the other hand, material and geometrical parameters are playing an important role when determining the noise contributions and the effect on the evaluation of $D^{*}$. Some of the parameters involved in the definitions of the noise contributions, as conductance, emissivity, or temperature of the device, are difficult to establish because of the subwavelength size of the elements, and because they are actually maps or distributions along the resonant element.

\section{ACKNOWLEDGMENTS}

This work has been supported by the Ministerio de Economia y Competitividad of Spain through projects TEC2013-40442 and TEC2012-38901-C02-01. F. J. Gonzalez would like to acknowledge support by project 32 of Centro Mexicano de Innovación en Energía Solar from Fondo Sectorial CONACYT-Secretaría de EnergíaSustentabilidad Energética and by the National Laboratory program from CONACYT through the Terahertz Science and Technology National Lab (LANCYTT)

\section{REFERENCES}

[1] Bharadwaj, P., Deutsch, B., and Novotny, L., "Optical antennas," Adv. Opt. Photon 1, 438-483 (2009).

[2] Novotny, L. and van Hulst, N., "Antennas for light," Nature Photonics 5, 83-90 (2011).

[3] Alda, J., Rico-García, J. M., López-Alonso, J. M., and Boreman, G., "Optical antennas for nano-photonic applications," Nanotechnology 16(5), S230 (2005).

[4] Puscasu, I., Schaich, W., and Boreman, G., "Resonant enhancement of emission and abosrption using frequency selective surfaces in the infrared," Infrared Physics and Technology 43(2), 101-107 (2002).

[5] Tharp, J., Lopez-Alonso, J. M., Ginn, J., Middleton, C., Lail, B., Munk, B. A., and Boreman, G., "Demonstration of a single-layer meanderline phase retarder at infrared," Optics Letters 31(18), 2687-2689 (2006).

[6] Ginn, J., Lail, B., Alda, J., and Boreman, G., "Planar infrared binary phase reflectarray," Optics Letters 33(8), 779-781 (2008). 
[7] Yu, N., Genevet, P., Aieta, F., Kats, M. A., Blanchard, R., Aoust, G., Tetienne, J. P., Gaburro, Z., and Capasso, F., "Flat optics: Controlling wavefronts with optical antenna metasurfaces," IEEE Journal of Selected Topics in Quantum Electronics 19(3), 4700423 (2013).

[8] Yu, N. and Capasso, F., "Flat optics with designer metasurfaces," Nature Materials 13, 139-150 (2014).

[9] Munk, B. A., [Frequency Selective Surfaces: Theory and Design], John Wiley and Sons (2000).

[10] Young, L., Robinson, L. A., and A., H. C., "Meander-line polarizer," IEEE Transaction on Antennas and Propagation 21(3), 376-378 (1973).

[11] Huang, J. and Encinar, J. A., [Reflectarrays Antennas], John Wiley and Sons (2008).

[12] Fumeaux, C., Herrmann, W., Kneubühl, F. K., and Rothouizen, H., "Nanometer thin-film ni-nio-ni diodes for detection and mixing of 30 thz radiation," Infrared Physics and Technology 39, 123-183 (1998).

[13] Codreanu, I. and Boreman, G., "Integration of microbolometers with infrared microstrip antennas," Infrared Physics and Technology 43, 334-344 (2002).

[14] Briones, E., Cuadrado, A., Briones, J., de Leon, R. D., Martinez-Anton, J. C., MacMurtry, S., Hehn, M., Montaigne, F., Alda, J., and Gonzalez, F. J., "Seebeck nanoantenas for the detection and characterization of infrared radiation," Optics Express 22(106), A1538-A1546 (2014).

[15] Lopez-Alonso, J. M., Mandviwala, T., Alda, J., and Boreman, G., "Infrared antenna metrology," in [Electrooptical and infrared systems: technology and applications II], Driggers, R., ed., 5987, 59870L1-11, SPIE (2005).

[16] Gonzalez, F. J., Fumeaux, C., Alda, J., and Boreman, G. D., "Thermal-impedance model of electrostatic discharge effects on microbolometers," Microwave and Optical Technology Letters 26, 291-293 (2000).

[17] Tang, L., Kocabas, S. E., Latif, S., A., O., Ly-Gagnon, D.-S., Saraswat, K., and Miller, D., "Nanometrescale germanium photodetector enhanced by a near-infrared dipole antenna," Nature Photonics 2, 226-229 (2008).

[18] Alda, J., Fumeaux, C., Codreanu, I., Schaefer, J., and Boreman, G., "Deconvolutoin method for twodimensional spatial-response mapping of lithographic infrared antennas," Applied Optics 38(19), 3993-4000 (1999).

[19] Rico-García, J. M., [Herramientas para el analisis y diseño de antenas ópticas], University Complutense of Madrid. PhD Dissertation (2007).

[20] Tucker, E., D'Archangel, J., Raschke, M., Briones, E., Gonzalez, F. J., and Boreman, G., "Near-field mapping of dipole nano-antenna-coupled bolometers," Journal of Applied Physics 114, 033109 (2013).

[21] Dereniak, E. and Boreman, G., [Infrared detectors and systems], John Wiley and Sons (1996).

[22] Cuadrado, A., Alda, J., and Gonzalez, F. J., "Multihpysics simulation for the optimization of optical nanoantennas working as distributed bolometers in the infrared," Journal of Nanophotonics 7, 07093 (2013).

[23] Fumeaux, C., Boreman, G., Herrmann, W., Keubuhl, F., and Rothuizen, H., "Spatial impulse response of lithographic infrared antennas," Applied Optics 38(1), 37-46 (1999). 